Research Paper

\title{
Up-regulation of LINC00467 promotes the tumourigenesis in colorectal cancer
}

\author{
Xiaoyun $\mathrm{He}^{1,2}$, Shen $\mathrm{Li}^{1}$, Bingbing $\mathrm{Yu}^{3}$, Gaoyan Kuang, ${ }^{4}$ Yongrong $\mathrm{Wu}^{4}$, Meili Zhang 3 , Yuxiang $\mathrm{He}^{5}$, \\ Chunlin $\mathrm{Ou}^{1,7, * \varpi}$, Pengfei Cao ${ }^{6,7}, * \varpi$
}

1. Department of Pathology, Xiangya Hospital, Central South University, Changsha, Hunan 410008, China.

2. Department of Endocrinology, Xiangya Hospital, Central South University, Changsha, Hunan 410008, China.

3. Department of Pathology, Dezhou People's Hospital, Dezhou, Shandong 253056, China

4. Department of Orthopedics, The First Affiliated Hospital of Hunan University of Chinese Medicine, Changsha, Hunan 410007, China.

5. Department of Oncology, Xiangya Hospital, Central South University, Changsha, Hunan 410008, China.

6. Department of Hematology, Xiangya hospital, Central South University, Changsha, Hunan 410008, China.

7. National Clinical Research Center for Geriatric Disorders, Xiangya Hospital, Central South University, Changsha 410008, Hunan, China.

*These authors jointly supervised this work.

$\square$ Corresponding author: Chunlin Ou. Department of Pathology, Xiangya Hospital, Central South University, Changsha, Hunan, 410008, China. Email: ouchunlin@csu.edu.cn; Pengfei Cao. Department of Hematology, Xiangya hospital, Central South University, Changsha, Hunan 410008, China. Email: caopengfei66@163.com.

(1) The author(s). This is an open access article distributed under the terms of the Creative Commons Attribution License (https://creativecommons.org/licenses/by/4.0/). See http://ivyspring.com/terms for full terms and conditions.

Received: 2019.07.19; Accepted: 2019.09.20; Published: 2019.10.19

\begin{abstract}
Recent studies have reported that long non-coding RNAs (IncRNAs) are associated with the tumourigenesis of colorectal cancer (CRC); however, several of these are yet to be identified and characterised. In this study, we report a novel IncRNA, LINC00467, which was significantly up-regulated in CRC; we investigated its function and mechanism in CRC. Our study demonstrated that LINC00467 levels in 45 pairs of CRC tissues were higher than those in the corresponding normal colon mucosal tissues. We used the Gene Expression Omnibus (GEO) and Gene Expression Profiling Interactive Analysis (GEPIA) databases for the analysis and measurement of clinical samples, and observed that the CRC patients with high LINC00467 expression levels showed poor overall survival (OS) and recurrent-free survival (RFS) rates. Furthermore, following the short interfering RNA (siRNA) knockdown of LINC00467 in the CRC cell line, the results demonstrated that LINC00467 suppresses the proliferation, invasion and metastasis of CRC cells in vitro. Moreover, its molecular mechanism of LINC00467 decreased the expression of Cyclin D1, Cyclin A1, CDK2, CDK4 and Twistl as well as enhanced the expression of E-cadherin. Collectively, these findings suggest that LINC00467 may be crucial in the progression and development of CRC, and may serve as a potential therapeutic target for CRC patients.
\end{abstract}

Key words: LINC00467, colorectal cancer, tumorigenesis, invasion; survival

\section{Introduction}

Colorectal cancer (CRC) is the second leading cause of cancer-related mortalities in the United States and the third most commonly diagnosed malignancy worldwide [1-4]. In the past decades, improvements in the therapeutic approach to $C R C$, have led to substantial progress in treating CRC. However, CRC-associated mortality has not changed as expected $[5,6]$. The progression and development of CRC may involve a multi-step process, majorly including the inherited and environmental factors that eventually result in a series of gene mutagenesis events associated with cancer cell apoptosis, proliferation, and differentiation $[7,8]$. With the popularity of gene tests and molecular therapy, several studies have investigated the molecular pathogenesis of CRC by analysing the molecular abnormalities in CRC progression and development [9-11].

Long non-coding RNAs (lncRNAs) form a subset of RNAs. Their transcribing length is more than 200 nucleotides (nt), and they lack a functional open reading frame (ORF); however, rarely, they may encode a functional short peptide $[12,13]$. Recently, lncRNAs have been widely studied due to the role they play in various human diseases, including cancer. Several studies revealed that lncRNAs were 
aberrantly expressed in various cancers, such as tongue squamous cell carcinoma (TSCC) [14], CRC [15], gastric cancer [16], cervical cancer [17] and hepatocellular cancer [18]. These abnormally expressed lncRNAs acted as biomarkers for cancer screening, analysis and therapy.

In this study, we analysed two previously published online datasets to find the dysregulated lncRNAs in CRC. One novel lncRNA, long inter-genic non-coding RNA 467 (LINC00467) was significantly overexpressed in the two CRC datasets. LINC00467 is located in the chr1q32.3 region, and measures $2616 \mathrm{nt}$ in length; however, little is known about the function and mechanism of LINC00467 in tumourigenesis. In this study, we determined that the LINC00467 was overexpressed in CRC tissues and cell lines, and via the Gene Expression Profiling Interactive Analysis (GEPIA) database, we observed that CRC patients with high LINC00467 expression levels demonstrated poor overall survival (OS) and recurrent-free survival (RFS). Furthermore, LINC00467 knockdown inhibits the proliferation and metastasis of colorectal cancer cells in vitro. Collectively, these results suggest that LINC00467, as a potential prognostic biomarker, is crucial in CRC progression and development.

\section{Materials and methods}

\section{Tissue samples and Bioinformatic analyses}

In total, 45 pairs of CRC tissues and corresponding normal colorectal tissues were collected from newly diagnosed CRC patients at the Xiangya Hospital of Central South University in Changsha, Hunan of China. None of these patients received routine radiotherapy. This study was approved by the Research Ethics Board of Xiangya Hospital of Central South University, and signed informed consent was obtained from each participant before they were enrolled in the study.

All microarray expression data containing primary colorectal cancer data and their correlated clinical data, were deposited in the Gene Expression Omnibus (GEO) database: GSE22598 [19], GSE37364 [20], and GSE50760 [21] (Affymetrix Human Genome U133 Plus 2.0 platform). GSE22598 contained 17 pairs of CRC and adjacent non-tumour tissues; GSE37364 had 38 normal colon samples and 56 primary CRC samples; GSE50760 had 18 metastasis CRC samples and adjacent non-metastasis samples.

\section{Cell culture and transfection}

The human normal colon mucosal cell line NCM460 and human colorectal cancer cell lines(HT29, SW480, SW620, HCT116) used in this study were obtained from the American Type Culture Collection (ATCC, Manassas, USA). HCT116 cells were maintained in DMEM (Dulbecco's Modified Eagle's medium) with $10 \%$ fetal bovine serum (FBS, Invitrogen, USA), and other cell lines (NCM460, SW480, SW620, HT29) were cultured in RPMI-1640 media (Invitrogen, USA) supplemented with 10\% FBS. When cell densities were about $60 \%$, 50nM siRNA oligos were transfected by Lipofectamine 3000 (Invitrogen, USA), according to the manufacturer's instructions. The sequences of the LINC00467 targeting siRNAs were: LINC00467-si-1: 5'GAAGAAGAGAAGAGAGAAA-3'; LINC00467-si-2: 5' - -GATAAGAAGTCCACTCACA-3'; sequences of non-target scramble controls were provided by RiboBio(Guangzhou, China).

\section{Quantitative real-time PCR}

RNA isolation and amplification were performed as described previously [22]. Thereafter, RNA was reverse transcribed to cDNA via a Revert Aid First Strand cDNA Synthesis kit (Fermentas, Canada). Quantitative real-time PCR (qRT-PCR) was performed using a SYBR_Premix ExTaq II kit (Takara, China) and CFX96 Real-Time PCR Detection System (Bio-Rad, USA) in order to determine the relative expression levels of the target genes. The qRT-PCR primer sequences are shown in Table 1.

Table 1. Primer sequence for real-Time PCR

\begin{tabular}{lll}
\hline Gene & Primer (Forward) & Primer (Reverse) \\
\hline $\begin{array}{l}\text { LINC0046 } \\
7\end{array}$ & ATTGAAGATGCTGCCAAGGG & GCCCAGTTCAGTCCCTCTT \\
CyclinD1 & TCGTTGCCCTCTGTGCCACA & GCAGTCCGGGTCACACTTGA \\
CyclinA1 & GAGGTCCCGATGCTTGTCAG & GTTAGCAGCCCTAGCACTGTC \\
CDK2 & AGCTGTGGACATCTGGAGCCT & CCCAACCTTGTGATGCAGCCA \\
& G & \\
CDK4 & TTGGTGTCGGTGCCTATGGG & CCATCAGCCGGACAACATTG \\
& & G \\
E-cadherin & TGAAGCCCCCATCTTTGTGC & GGCTGTGTACGTGCTGTTCT \\
Twist1 & CAGCGCACCCAGTCGCTGAA & CCAGGCCCCCTCCATCCTCC \\
U6 & GTGGGCCGAAGATATGCTCA & TTGGCTAGGACCTGGCTGTA \\
GAPDH & CTCGCTTCGGCAGCACA & AACGCTTCACGAATTGCGT \\
\hline
\end{tabular}

\section{Subcellular Fractionation Analysis}

To determine the cellular localization of LINC00467, cytoplasmic and nuclear RNA were separated and collected via the PARIS Kit (Invitrogen, USA), according to the manufacturer's instructions. The computing method was used as described previously [23].

\section{Western blotting}

Lysis, electrophoresis, and target protein visualisation were performed as described previously [22]. Briefly, the cell lysates $(50 \mu \mathrm{g})$ were separated by $10 \%$ sodium dodecyl sulphate-polyacrylamide gel electrophoresis (SDS-PAGE) and were then transferred onto a polyvinylidene fluoride (PVDF) 
membrane. Thereafter, the membranes were blocked in $5 \%$ defatted milk for $1 \mathrm{~h}$, and incubated overnight at $4{ }^{\circ} \mathrm{C}$ with primary anti-cyclinD1, CDK4, Twist1, E-cadherin and GAPDH antibodies (Cell Signalling Technology, Danvers, MA, USA). The next day, the membranes were incubated with horseradish peroxidase-conjugated secondary antibodies (Santa Cruz Biotechnology) for $1 \mathrm{~h}$ at room temperature. The signal was visualised via the ECL detection system, and densitometric analysis of the immunodetected bands was carried out using Image $\mathrm{J}$ software (http://rsb.info. nih.gov/ij).

\section{Cell proliferation assay}

Cell proliferation assay was carried out using CCK-8 (Dojin Laboratories, Japan), as described previously [24]. Each experiment was repeated thrice independently.

\section{Flow cytometry for cell cycle analysis}

After transfecting with si-NC or si-LINC00467 for $48 \mathrm{~h}$, approximately $1 \times 10^{6}$ HT29 cells were collected for cell cycle distribution, and the DNA content was detected using propidium iodide (PI) (Sigma, San Antonio, USA) staining, according to the methods described in a previous study [25]. Cell cycle distribution was analysed via flow cytometry (Beckman Coulter, South Kraemer, USA) using cell Modifit software. Each experiment was repeated thrice independently.

\section{Transwell Matrigel assays}

The invasiveness of the CRC cells was determined by an assay performed in a 24 well Transwell plate ( $8 \mu \mathrm{M}$ pore size; Costar), as described previously [26]. Briefly, $5 \times 10^{4}$ cells were placed on the upper chamber of each insert coated with 200 $\mathrm{mg} / \mathrm{ml}$ of Matrigel (BD Biosciences, CA, USA). After $48 \mathrm{~h}$, the invaded cells were stained with haematoxylin and enumerated. Each experiment was repeated thrice independently.

\section{Statistical analysis}

All statistical analyses were carried out using the SPSS version 18.0 software and GraphPad prism version 6.0. Data are presented as mean \pm standard error of mean. Differences between the two independent groups were tested with the Student's t-test. OS and RFS were calculated using the Kaplan-Meier method, and the results of a log-rank test were considered significant if $p<0.05$.

\section{Results}

\section{LINC00467 is highly expressed in the CRC tissues and cell lines}

To find the dysregulated lncRNAs in CRC, two online GEO datasets (\#GSE22598 and GSE37364) based on the Affymetrix Human Genome U133 Plus 2.0 platform were analysed. One novel lncRNA LINC00467 was significantly up-regulated in the CRC tissues compared with the non-tumour tissues according to the GSE22598 and GSE37364 datasets ( $p$ $<0.05$, Fig. 1A and B). Furthermore, we detected LINC00467 expression in 45 pairs of CRC and adjacent non-tumour tissues, and found that the LINC00467 expression was higher in CRC tissues than in adjacent non-tumour tissues $(p<0.05$, Fig. 1C). Next, LINC00467 expression levels were also determined by qRT-PCR in four CRC cell lines (SW480, HT29, HCT116 and SW620) and the normal colon mucosal cell line NCM460. The results revealed that LINC00467 expression was higher inCRC cell lines than in NCM460 ( $p<0.05$, Fig. 1D), and that it was the highest in HT29 cells. Moreover, we analysed the distribution proportion of LINC00467 expression in the nucleus and cytoplasm, and found that LINC00467 expression was higher in the cytoplasm than the nucleus, indicating that subcellular localization of LINC00467 in CRC cells was majorly located in the cytoplasm (Fig. 1E).

\section{LINC00467 up-regulation is associated with poor prognosis in CRC}

We next assessed the correlation of LINC00467 expression with the clinicopathological features of CRC, wherein we assessed the correlation between LINC00467 expression and distant metastasis in CRC tissues, which was previously analysed by one published dataset (\#GSE50760) using Affymetrix HG_U133 Plus 2 arrays. The analysis indicated that higher expression of LINC00467 significantly correlated with distant metastasis in CRC $(p=0.004$, Fig. 2A). Thereafter, by accessing the Cancer RNA-Seq Nexus (CRN) database [27], we found that LINC00467 expression in the pathological stages (I-IV) of CRC was higher than that observed in normal tissues (Table 2). By analysing the public CRC datasets in the GEPIA database [28], we found that LINC00467 was significantly up-regulated in the colon and rectal adenocarcinoma samples (all $p<0.05$, Fig. 2B), and that high LINC00467 expression was associated with poor RFS and OS (all $p<0.05$, Fig. 2C and D). Collectively, these data indicate that high LINC00467 expression levels are an independent risk factor for CRC patients. 
A

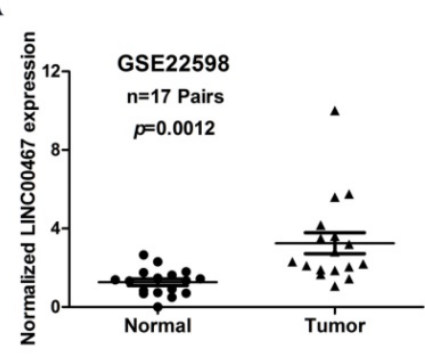

C

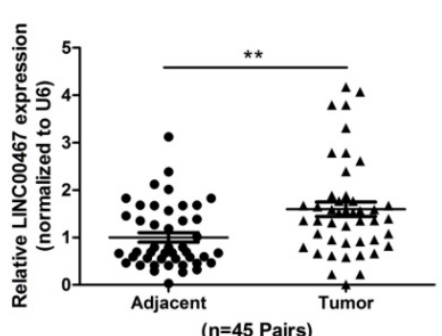

$\mathbf{E}$

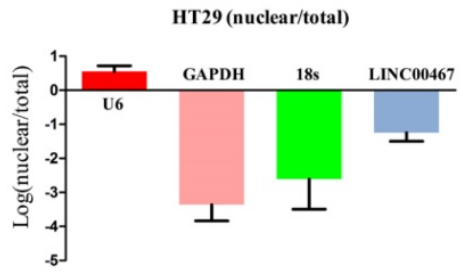

B

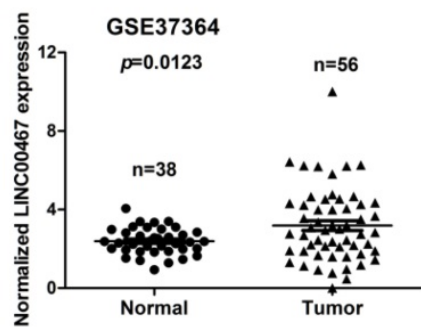

D

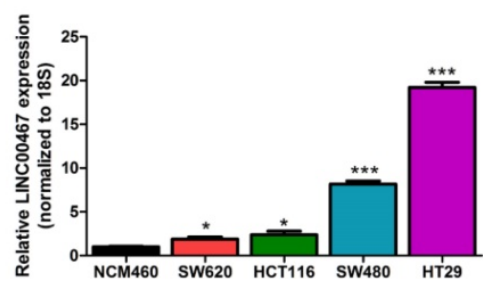

Figure 1. LINC00467 expression is up-regulated in CRC tissues and cell lines. (A) \#GSE22598 (containing 17 pairs of CRC tissues and corresponding normal colorectal tissues) and (B) \#GSE37364 (38 normal colon samples and 56 primary CRC samples) from the GEO database were used to analyse the expression of LINC00467. (C) LINC00467 is highly expressed in 45 pairs of CRC tissues compared with the corresponding normal colorectal tissues. (D) LINC00467 expression was significantly increased in the CRC cell lines (SW480, SW620, HT29 and HCT116) compared with NCM460, a normal colon mucosal cell line. (E and F) Nucleic and cytoplasmic RNA were analysed using qRT-PCR to detect the expression levels of LINC00467 in HT29 and SW480 cells. U6 was used as a nucleic RNA control; GAPDH and $\beta$-actin were used as cytoplasmic RNA controls. Data are presented as mean \pm SEM. $* p<0.05$ compared with control.

A

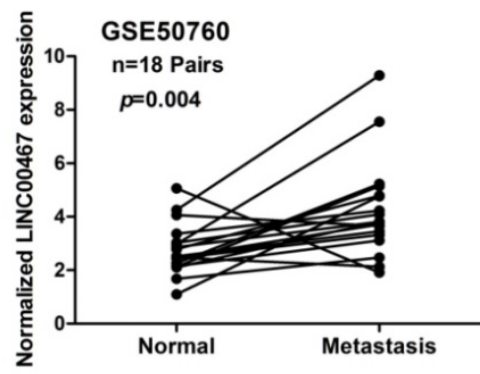

C

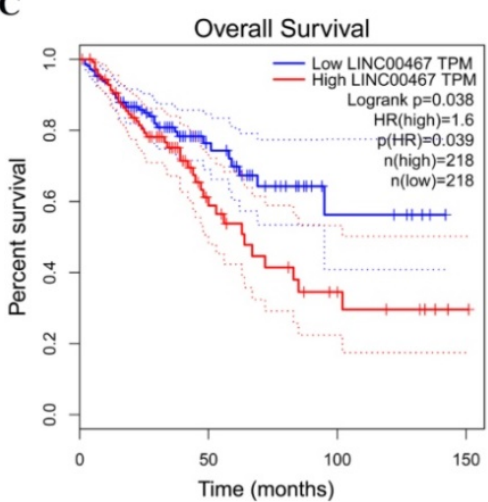

B

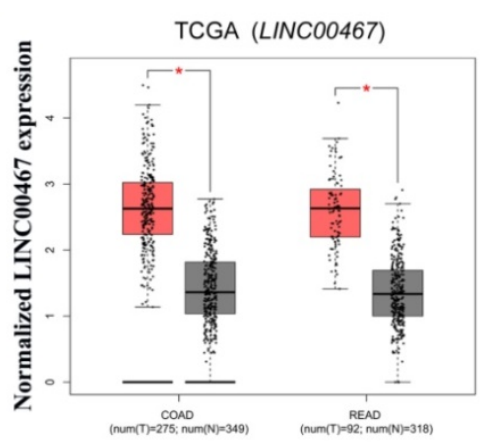

D

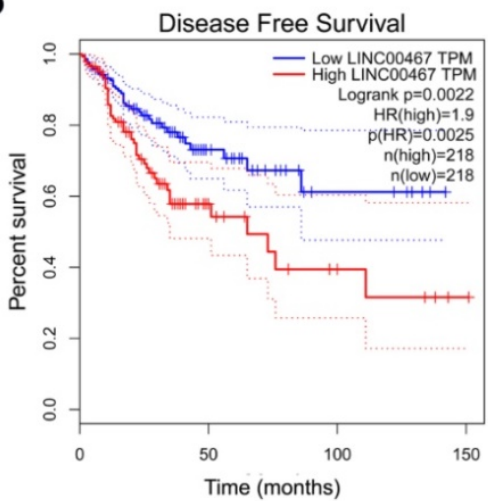

Figure 2. Relationship between LINC00467 expression and clinicopathological features. (A) Relative expression of LINC00467 in normal and metastatic human CRC tissues from the GEO database (\#GSE50760, 18 metastasis CRC samples and adjacent non-metastatic samples) was studied. (B) The GEPIA database was used to analyse LINC00467 expression in the CRC samples (COAD stands for colon adenocarcinoma and READ for rectal adenocarcinoma). (C-D) GEPIA database was used to analyse the clinical impact of LINC00467 expression patterns on the CRC patients' RFS and OS (the TCGA CRC specimens were divided into two groups within the GEPIA database, group 1 = low LINC00467 expression, $n=218$; group $2=$ high LINC00467 expression, $n=218)$. ${ }^{p}<0.05$ compared with control. 
A

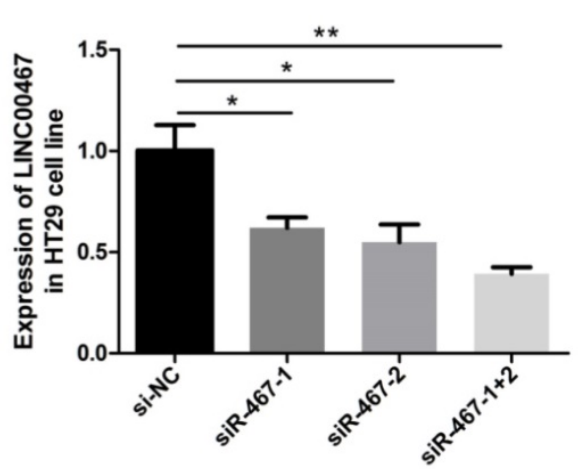

C

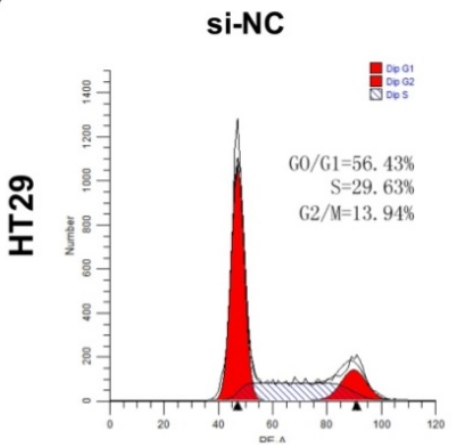

siR-467
B

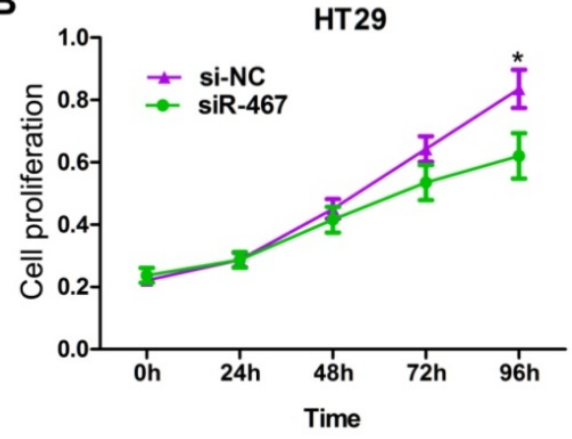

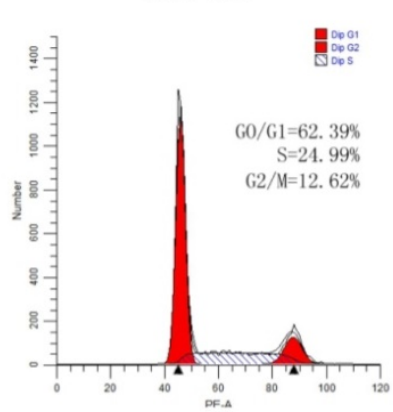

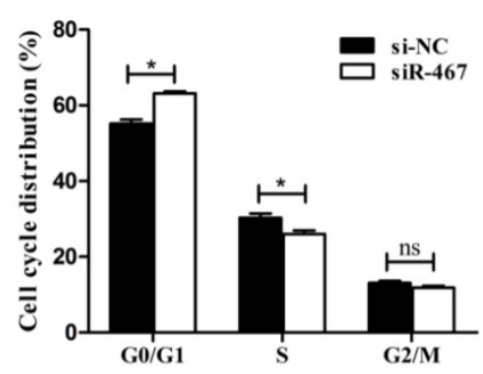

Figure 3. Knockdown of LINC00467 expression impeded the proliferation of CRC cells. (A) The interference efficiency of siR-467 was verified in HT29 cells. HT29 cells were transfected with either si-NC or siR-467 (1\#, 2\#, 1+2\#) for $48 \mathrm{~h}$, and then, LINC00467 expression was analysed by qRT-PCR. After transfecting HT29 cells with si-NC or siR-467 for $48 \mathrm{~h}$, the CCK8 assay (B) and flow cytometry $(C)$ were used to detect the cell proliferative ability. Data are presented as mean \pm SEM. ${ }^{*} p<0.05$, $* * p<0.01$ compared with control.
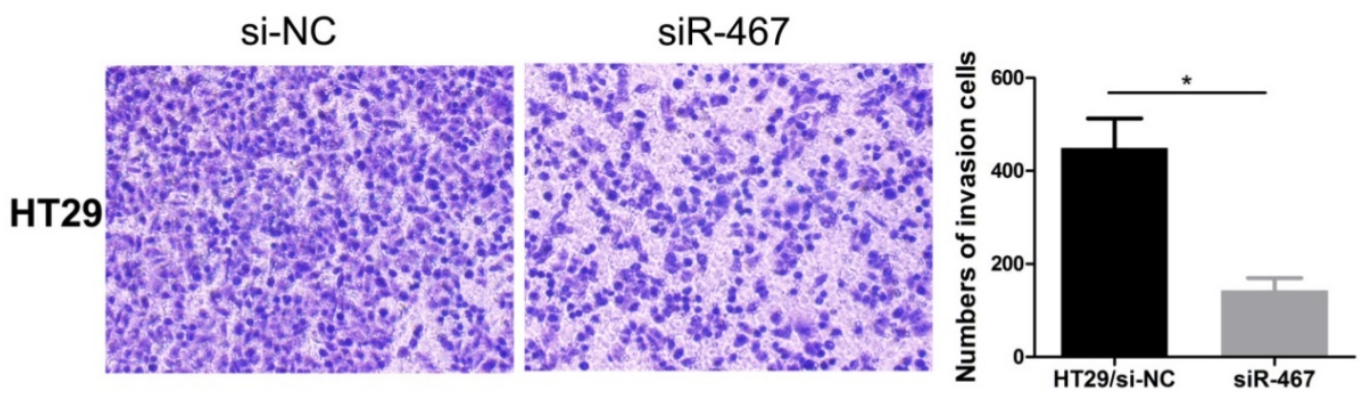

Figure 4. Knockdown of LINC00467 expression impeded the invasiveness of CRC cells. The invasive ability of HT29 cells was detected using a Transwell Matrigel assay after transfecting with si-NC or siR-467 for $48 \mathrm{~h}$. Data are presented as mean \pm SEM. *p $<0.05$ compared with control.

\section{Knockdown of LINC00467 expression inhibits cell proliferation and invasion in CRC}

To verify LINC00467 function in CRC cells, we first measured the efficiency of the short interfering RNA (siRNA), siRNA-LINC00467(siR-467). The results demonstrated that the siR-467-1+2 group, when compared with both siR-467-1 and siR-467-2 groups revealed the highest interfering efficiency in HT29 cells (Fig. 3A). Therefore, we transfected the siRNA-LINC00467-1+2 in HT29 cells to detect and analyse the change in biological function. After investigating the siRNA efficacy, we assessed the biological function induced by LINC00467 knockdown in the CRC cells. Initially, we explored the effect of LINC00467 knockdown on the proliferation of CRC cells. By performing CCK-8 proliferation assays, we found that knocking down LINC00467 expression significantly inhibited HT29 cell proliferation relative to that of the control cells ( $p$ $<0.05$, Fig. 3B). Meanwhile, flow cytometry analysis revealed that knocking down LINC00467 expression in HT29 cells elevated the percentage of cells in the G1 phase and reduced the percentage of cells in the $S$ phase $(p<0.05$, Fig. 3C).

Subsequently, we also explored the effect of LINC00467 knockdown on the invasion of CRC cells. We detected the effect of LINC00467 on the invasion of CRC cells using a Transwell Matrigel assay. The results indicated that knocking down LINC00467 expression significantly inhibited the invasion capacity of HT29 cells compared to the control group $(p<0.05$, Fig. 4). 
A

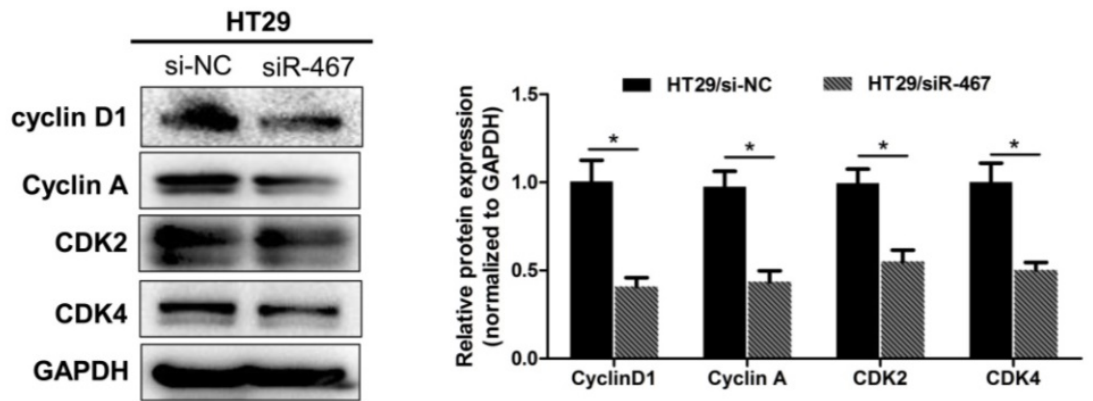

B

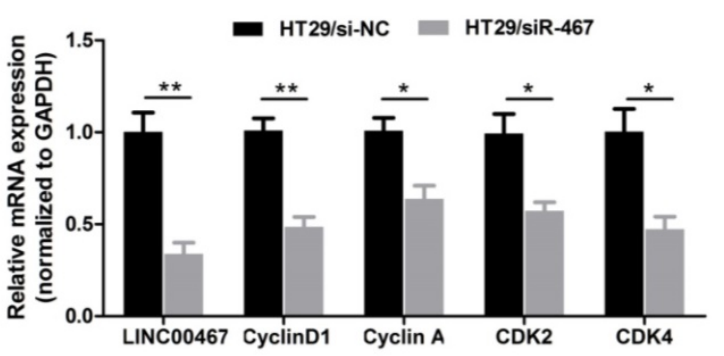

C
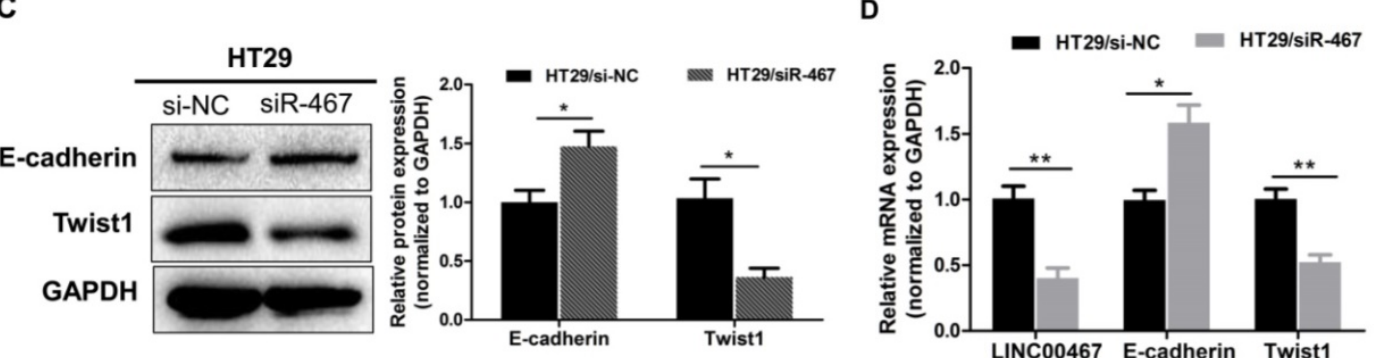

Figure 5. LINC00467 knockdown inhibits the proliferation and EMT markers expressed in CRC. After transfecting with si-NC or siR-467 for $48 \mathrm{~h}$, the effect of LINC00467 on the protein and mRNA expressions of Cyclin DI, Cyclin A1, CDK2 and CDK4 (A-B) and of E-cadherin and Twistl (C-D) in HT29 cells were analysed by western blotting, densitometry and qRT-PCR. Data are presented as the mean \pm SEM. ${ }^{*} p<0.05$, ${ }^{*} p<<0.01$ compared with the control.

Table 2. The expression of LINC00467 in TCGA Colon adenocarcinoma (COAD) RNA-seq dataset were analyzed by the Cancer RNASeq Nexus.

\begin{tabular}{lll}
\hline & LINC00467 (Transcript ID: uc001hil.3) \\
\hline Colon adenocarcinoma subset pair & Average expression in cancer & Average expression in normal \\
\hline Colon adenocarcinoma--Stage I versus Normal (adjacent normal) & 8.00 & 5.06 \\
Colon adenocarcinoma--Stage II versus Normal (adjacent normal) & 6.75 & 5.06 \\
Colon adenocarcinoma--Stage IIA versus Normal (adjacent normal) & 8.18 & 5.06 \\
Colon adenocarcinoma--Stage IIB versus Normal (adjacent normal) & 11.83 & 5.06 \\
Colon adenocarcinoma--Stage III versus Normal (adjacent normal) & 6.15 & 5.06 \\
Colon adenocarcinoma--Stage IIIA versus Normal (adjacent normal) & 7.22 & 5.06 \\
Colon adenocarcinoma--Stage IIIB versus Normal (adjacent normal) & 8.88 & 5.06 \\
Colon adenocarcinoma--Stage IIIC versus Normal (adjacent normal) & 8.43 & 5.06 \\
Colon adenocarcinoma--Stage IV versus Normal (adjacent normal) & 7.95 & 5.06
\end{tabular}

Note: The Cancer RNASeq Nexus (CRN, http://syslab4.nchu.edu.tw/CRN) is an open resource for intuitive data exploration, providing coding-transcript/lncRNA

expression profiles that contain alternative splicing to support researchers generating new hypotheses in cancer research and personalized medicine.

\section{Knockdown of LINC00467 expression regulates proliferated and epithelial- mesenchymal transition (EMT) markers in CRC}

To further decipher the molecular mechanism by which knocking down LINC00467 expression suppressed the proliferation and invasion of CRC cells in vitro, we assessed the mRNA and protein level of the proliferated markers Cyclin D1, Cyclin A1, CDK2 and CDK4, as well as, the epithelial marker Ecadherin, and the mesenchymal markers Twist1 in HT29 cell lines, using qRT-PCR and western blotting techniques. Knocking down LINC00467 significantly inhibited the expression of the proliferated markers Cyclin D1, Cyclin A1, CDK2 and CDK4 $(p<0.05$, Fig. 5A-B). Meanwhile, LINC00467 knockdown significantly reduced the expression of mesenchymal 
markers Twist1 and enhanced the expression of the epithelial marker E-cadherin ( $p<0.05$, Fig. 5C-D), thereby suppressing progression of epithelial-mesenchymal transition (EMT). Moreover, by analysing the GSE37364 datasets, we found that cyclin D1, CDK4, and Twist1 were significantly up-regulated in CRC tissues, whereas E-cadherin was significantly down-regulated in CRC tissues when compared with the non-tumour tissues (all $p<0.05$, Supplemental Fig. 1). These results indicated that LINC00467 may contribute to regulating the expression of proliferated and EMT marker expression in the CRC cells.

\section{Discussion}

CRC is the third most prevalent cancer worldwide and it substantially affects human health [29]. In 2015, 777,987 new cases and 352,589 deaths occurred due to CRC [30]. With the development of basic and clinical investigations, the mechanisms of CRC and therapeutic strategies involved have become better understood [31]. Nevertheless, due to postsurgical recurrence and metastasis of primary tumours, CRC mortality and morbidity rates remain high. Therefore, it is important to identify novel molecular therapeutic targets for CRC diagnosis, screening and therapy.

Recently, substantial evidence has demonstrated that $\operatorname{lncRNAs}$ are crucial in regulating various biological processes by modulating gene expression at the epigenetic, transcriptional and posttranscriptional levels $[12,32]$, thereby impacting the progression and development of several diseases, particularly cancer. Recent studies report that the disruption or disabling of IncRNAs levels closely correlates with the cancer cell proliferation and apoptosis, epithelialmesenchymal transition (EMT) and drug resistance [33-36]. Meanwhile, IncRNAs, including MALAT1 [37], HOTAIR [38], GAS5 [39], NEAT1 [40], and TUG1 [18], have been demonstrated to be differentially expressed and indicate poor prognosis. IncRNAs are considered as a kind of deal biomarker for tumour diagnosis and tumour recurrence surveys because they detect with high specificity and sensitivity, are easier to extract and exist steadily in blood and tissue [41].

With the popularity of gene microarray technologies and high-throughput sequencing, an increasing number of public databases (eg, TCGA, Oncomine, GEO, etc) have become powerful tools for predicting and identifying valuable lncRNAs [15]. In this study, we analysed the lncRNAs that were found to be dysregulated in CRCs in previously published online GEO datasets (\#GSE22598 and GSE37364), based on the Affymetrix Human Genome U133 Plus 2.0 platform, and found one novel lncRNA (LINC00467), which was significantly overexpressed in the two CRC datasets. LINC00467 is located in the chr1q32.3 region and is $2616 \mathrm{nt}$ long, and has only been previously reported to be found in the neuroblastoma [42]. In our study, we aimed to explore the properties of LINC00467 as a novel biomarker for CRC diagnosis and prognosis. We report here that LINC00467 expression in CRC tissues was significantly higher than those in the matched adjacent normal tissues. Meanwhile, LINC00467 overexpression in CRC patients had poorer RFS and OS rates, which may be an independent poor prognostic factor for the CRC patients, as suggested by multivariate analysis results.

Moreover, the biological functions and molecular mechanism of LINC00467 have been seldom reported. Only one study by Atmadibrata [42] reported that in neuroblastoma, N-Myc targeting LINC00467 could reduce the expression of tumour suppressor gene Dickkopf-related protein 1 (DKK1), thereby promoting neuroblastoma cell survival [42]. However, the effect of LINC00467 on the tumourigenesis of other tumours is poorly understood. In this study, our results demonstrate that knocking down LINC00467 in HT29 cell lines could suppress the proliferation and invasiveness of CRC cells. Furthermore, we have shown that subcellular localization of LINC00467 in the CRC cells was majorly located at the cytoplasm. Subcellular localization of lncRNAs is usually related to their biological function [43]. Therefore, we speculated that the cytoplasmic expression of LINC00467 in the CRC cells may sponge with miRNA to form the ceRNA, thereby regulating the malignant biological behaviour of CRC. In our next study, we will further explore the regulatory mechanism of LINC00467 in CRC progression.

In conclusion, we found that a novel lncRNA, LINC00467, was the most significantly up-regulated lncRNA in CRC. Elevated LINC00467 expression was associated with poor survival time (RFS and OS), and multivariate analysis results indicated that a high LINC00467 expression level is an independent risk factor for CRC patients. We also demonstrated that knockdown of LINC00467 could mediate the proliferation and invasion of CRC cells, thereby indicating that further investigation and study of LINC00467 may lead to the development of novel CRC therapies.

\section{Supplementary Material}

Supplementary figure $\mathrm{S} 1$.

http://www.jcancer.org/v10p6405s1.pdf 


\section{Acknowledgements}

This study was supported by the National Natural Science Foundation of China (81903032), the Natural Science Foundation of Hunan Province of China (2019JJ40487 and 2019JJ40497), the Postgraduate Research and Innovation Project of Hunan Province (CX2018B115), the student innovation project of Central south university (2018zzts044) and the Youth Fund of Xiangya Hospital (2018Q011).

\section{Competing Interests}

The authors have declared that no competing interest exists.

\section{References}

1. Siegel RL, Miller KD, Fedewa SA, Ahnen DJ, Meester RGS, Barzi A, et al. Colorectal cancer statistics. 2017. CA Cancer J Clin. 2017; 67: 177-93.

2. Siegel RL, Miller KD, Jemal A. Cancer Statistics, 2017. CA Cancer J Clin. 2017; 67: $7-30$

3. Chen W, Zheng R, Baade PD, Zhang S, Zeng H, Bray F, et al. Cancer statistics in China, 2015. CA Cancer J Clin. 2016; 66: 1

4. Obuch JC, Ahnen DJ. Colorectal Cancer: Genetics is Changing Everything. Gastroenterol Clin North Am. 2016; 5: 459-76.

5. Walsh JM, Terdiman JP. Colorectal cancer screening: scientific review. Jama. 2003; 289: 1288-96.

6. Sunkara V, Hebert JR. The colorectal cancer mortality-to-incidence ratio as an indicator of global cancer screening and care. Cancer-am Cancer Soc. 2015; 121: 1563-69.

7. Ren W, Shen S, Sun Z, Shu P, Shen X, Bu C, et al. Jak-STAT3 pathway triggers DICER1 for proteasomal degradation by ubiquitin ligase complex of CUL4A(DCAF1) to promote colon cancer development. Cancer Lett. 2016; 375: 209-20.

8. Calver PM, Frucht $\mathrm{H}$. The genetics of colorectal cancer. ANN INTERN MED. 2002; 137: 603-12.

9. Yiu AJ, Yiu CY. Biomarkers in Colorectal Cancer. Anticancer Res. 2016; 36: 1093-102.

10. ColussiI D, Brandi G, Bazzoli F, Ricciardiello L. Molecular pathways involved in colorectal cancer: implications for disease behavior and prevention. Int J Mol Sci. 2013;14:16365-85

11. Tang A, Li N, Li X, Yang H, Wang W, Zhang L, et al. Dynamic activation of the key pathways: linking colitis to colorectal cancer in a mouse model. Carcinogenesis. 2012; 33:1375-83.

12. He X, Ou C, Xiao $Y$, Han $\mathrm{O}, \mathrm{Li} \mathrm{H}$, Zhou S. LncRNAs: key players and novel insights into diabetes mellitus. Oncotarget. 2017; 8: 71325-41.

13. Bazzini AA, Johnstone TG, Christiano R, Mackowiak SD, Obermayer B, Fleming ES, et al. Identification of small ORFs in vertebrates using ribosome footprinting and evolutionary conservation. Embo J. 2014; 33: 981-93.

14. Yu J, Liu Y, Gong Z, Zhang S, Guo C, Li X, et al. Overexpression long non-coding RNA LINC00673 is associated with poor prognosis and promotes invasion and metastasis in tongue squamous cell carcinoma. Oncotarget. 2017; 8:16621-32.

15. Wang X, Mo FM, Bo H, Xiao L, Chen GY, Zeng PW, et al. Upregulated Expression of Long Non-Coding RNA, LINC00460, Suppresses Proliferation of Colorectal Cancer. J Cancer. 2018; 9: 2834-43.

16. Song H, Sun W, Ye G, Ding G, Liu Z, Zhang S, et al. Long non-coding RNA expression profile in human gastric cancer and its clinical significances. J Transl Med. 2013; 11: 225.

17. Shang C, Zhu W, Liu T, Wang W, Huang G, Huang J, et al. Characterization of long non-coding RNA expression profiles in lymph node metastasis of early-stage cervical cancer. Oncol Rep. 2016; 35: 3185-97.

18. Peng L, Jiang B, Yuan X, Qiu Y, Peng J, Huang $Y$, et al. Super-Enhancer-Associated Long Noncoding RNA HCCL5 Is Activated by ZEB1 and Promotes the Malignancy of Hepatocellular Carcinoma. Cancer Res, 2019; 79:572-84.

19. Okazaki S, Ishikawa $T$, Iida $S$, Ishiguro $M$, Kobayashi $H$, Higuchi $T$, et al. Clinical significance of UNC5B expression in colorectal cancer. Int J Oncol. 2012; 40: 209-16.

20. Valcz G, Patai AV, Kalmar A, Peterfia B, Furi I, Wichmann B, et al. Myofibroblast-derived SFRP1 as potential inhibitor of colorectal carcinoma field effect. Plos One. 2014; 9: e106143.

21. Kim SK, Kim SY, Kim JH, Roh SA, Cho DH, Kim YS, et al. A nineteen gene-based risk score classifier predicts prognosis of colorectal cancer patients. Mol Oncol. 2014; 8: 1653-66.
22. Ou $\mathrm{C}$, Sun $\mathrm{Z}$, Zhang $\mathrm{H}$, Xiong $\mathrm{W}$, Ma J, Zhou $\mathrm{M}$, et al. SPLUNC1 reduces the inflammatory response of nasopharyngeal carcinoma cells infected with the EB virus by inhibiting the TLR9/NF-kappaB pathway. Oncol Rep. 2015; 33: 2779-88

23. Huarte M, Guttman M, Faldser D, Garber M, Koziol MJ, Kenzelmann-Broz D, et al. A large intergenic noncoding RNA induced by p53 mediates global gene repression in the 553 response. Cell. 2010; 142: 409-19.

24. Li Y, Zhao C, Yu Z, Chen J, She X, Li P, et al. Low expression of miR-381 is a favorite prognosis factor and enhances the chemosensitivity of osteosarcoma. Oncotarget. 2016; 7: 68585-96.

25. Dang W, Qin Z, Fan S, Wen Q, Lu Y, Wang J, et al. miR-1207-5p suppresses lung cancer growth and metastasis by targeting CSF1. Oncotarget. 2016; 7: 32421-32.

26. Ou C, Sun Z, Li X, Ren W, Oin Z, Zhang X, et al. MiR-590-5p, a density-sensitive microRNA, inhibits tumorigenesis by targeting YAP1 in colorectal cancer. Cancer Lett. 2017; 399: 53-63.

27. Li JR, Sun CH, Li W, Chao RF, Huang CC, Zhou XJ, et al. Cancer RNA-Seq Nexus: a database of phenotype-specific transcriptome profiling in cancer cells. Nucleic Acids Res. 2016; 44: D944-51.

28. Tang Z, Li C, Kang B, Gao G, Li C, Zhang Z. GEPIA: a web server for cancer and normal gene expression profiling and interactive analyses. Nucleic Acids Res. 2017; 45: W98-102.

29. Bray F, Ren JS, Masuyer E, Ferlay J. Global estimates of cancer prevalence for 27 sites in the adult population in 2008. Int J Cancer. 2013; 132: 1133-45.

30. Ferlay J, Shin HR, Bray F, Forman D, Mathers C, Parkin DM. Estimates of worldwide burden of cancer in 2008: GLOBOCAN 2008. Int J Cancer. 2010; 127: 2893-917.

31. Ou C, Sun Z, Li S, Li G, Li X, Ma J. Dual roles of yes-associated protein (YAP) in colorectal cancer. Oncotarget. 2017; 8: 75727-41.

32. Ponting $\mathrm{CP}$, et al.. Evolution and functions of long noncoding RNAs. Cell. 2009; 136: 629-41.

33. Schmitt AM, Chang HY. Long Noncoding RNAs in Cancer Pathways. Cancer Cell. 2016; 29: 452-63.

34. Fan C, Tang Y, Wang J, Xiong F, He Y, Wei F, et al. Role of long non-coding RNAs in glucose metabolism in cancer. Mol Cancer. 2017; 16: 130.

35. Zhong Y, Du Y, Yang X, Mo Y, Fan C, Xiong F, et al. Circular RNAs function as ceRNAs to regulate and control human cancer progression. Mol Cancer. 2018; 17:79.

36. Tang Y, He Y, Zhang P, Wang J, Fan C, Yang L, et al. LncRNAs regulate the cytoskeleton and related Rho/ROCK signaling in cancer metastasis. Mol Cancer. 2018; 17: 77.

37. He X, Yan Q, Kuang G, Wang Y, Cao P, Ou C. Metastasis-associated lung adenocarcinoma transcript 1 regulates tumor progression: old wine in a new bottle. J Thorac Dis. 2018; 10: S1088-91.

38. Wang $R$, Shi $Y$, Chen $L$, Jiang $Y$, Mao $C$, Yan B, et al. The ratio of FoxA1 to FoxA2 in lung adenocarcinoma is regulated by LncRNA HOTAIR and chromatin remodeling factor LSH. Sci Rep. 2015;5:17826.

39. Yin $\mathrm{D}$, He X, Zhang E, Kong R, De W, Zhang Z Long noncoding RNA GAS5 affects cell proliferation and predicts a poor prognosis in patients with colorectal cancer. Med Oncol. 2014; 31: 253.

40. Li Y, Li Y, Chen W, He F, Tan Z, Zheng J, et al. NEAT expression is associated with tumor recurrence and unfavorable prognosis in colorectal cancer. Oncotarget. 2015; 6: 27641-50.

41. Wang Y, Mo Y, Gong Z, Yang X, Yang M, Zhang S, et al. Circular RNAs in human cancer. Mol Cancer. 2017: 16: 25 .

42. Atmadibrata B, Liu PY, Sokolowski N, Zhang L, Wong M, Tee AE, et al. The novel long noncoding RNA linc00467 promotes cell survival but is down-regulated by N-Myc. Plos One. 2014; 9: e88112.

43. Chen LL. Linking Long Noncoding RNA Localization and Function. Trends Biochem Sci. 2016; 41: 761-72. 